\title{
Same-day discharge after appendectomy for acute appendicitis: a systematic review and meta-analysis
}

\author{
Elisabeth M. L. de Wijkerslooth ${ }^{1}$ (D) - Jay M. Bakas ${ }^{1}$ • Joost van Rosmalen ${ }^{2} \cdot$ Anne Loes van den Boom $^{1}$. \\ Bas P. L. Wijnhoven ${ }^{1}$
}

Accepted: 27 January 2021 / Published online: 11 February 2021

(C) The Author(s) 2021

\begin{abstract}
Purpose Patients presenting with acute appendicitis are usually hospitalized for a few days for appendectomy and postoperative recovery. Shortening length of stay may reduce costs and improve patient satisfaction. The purpose of this study was to assess the safety of same-day discharge after appendectomy for acute appendicitis.

Methods A systematic review was performed according to PRISMA guidelines. A literature search of EMBASE, Ovid MEDLINE, Web of Science, Cochrane Central, and Google Scholar was conducted from inception to April 14, 2020. Two reviewers independently screened the literature and selected studies that addressed discharge on the same calendar day as the appendectomy. Risk of bias was assessed with the ROBINS-I tool. Main outcomes were hospital readmission, complications, and unplanned hospital visits in the postoperative course. A random effects model was used to pool risk ratios for the main outcomes.

Results Of the 1912 articles screened, 17 comparative studies and 8 non-comparative studies met the inclusion criteria. Most only included laparoscopic procedure for uncomplicated appendicitis. Most studies were considered at moderate or serious risk of bias. In meta-analysis, same-day discharge (vs. overnight hospitalization) was not associated with increased rates of readmission, complication, and unplanned hospital visits. Non-comparative studies demonstrated low rates of readmission, complications, and unplanned hospital visits after same-day discharge.

Conclusion This study suggests that same-day discharge after laparoscopic appendectomy for uncomplicated appendicitis is safe without an increased risk of readmission, complications, or unplanned hospital visits. Hence, same-day discharge may be further encouraged in selected patients.

Trial registration PROSPERO registration no. CRD42018115948
\end{abstract}

Keywords Appendicitis $\cdot$ Appendectomy $\cdot$ Same-day discharge $\cdot$ Length of stay $\cdot$ Readmission

\section{Introduction}

Acute appendicitis is one of the most frequent surgical emergencies worldwide and is associated with a substantial clinical and financial burden. Appendectomy is mostly performed

Meeting presentation: A preliminary abstract was presented at the American College of Surgeons Clinical Congress, October 27-31, 2019. San Francisco, CA, USA.

Elisabeth M. L. de Wijkerslooth e.dewijkerslooth@erasmusmc.nl

1 Department of Surgery, Erasmus MC-University Medical Centre, PO Box 2040, 3000, CA Rotterdam, the Netherlands

2 Department of Biostatics, Erasmus MC-University Medical Centre, Rotterdam, the Netherlands through laparoscopy, enabling quick recovery of the patient. Reducing length of stay (LOS) may relieve pressure on hospital bed capacity, reduce healthcare costs, and improve treatment satisfaction [1-5]. Many studies have evaluated the safety and feasibility of expedited discharge after appendectomy. However, the terminology and definitions used for early discharge vary greatly [1-11]. Usually, outpatient appendectomy is defined as discharge after appendectomy without hospital admission and ambulatory appendectomy as postoperative LOS of $12 \mathrm{~h}$ at most (with or without overnight hospitalization) $[1,3]$. Day-case and same-day suggest discharge on the day of surgery, but are often defined as a maximum postoperative LOS of $24 \mathrm{~h}$ [2,12]. Criteria for patient selection and discharge vary as well. Most often only patients with laparoscopic procedure for simple appendicitis (without perforation or necrosis) are considered eligible for same-day discharge. 
Some studies also selected for patients without concerns of comorbidities or social/organizational contraindications. A recent review of five studies on ambulatory laparoscopic appendectomy among adults demonstrated its feasibility but the authors were concerned about the methodological quality of the included studies [13]. Several other studies have shown the feasibility of same-day discharge (SDD), defined as discharge on the same calendar day as appendectomy [5, 8, 9, 14, 15]. Nevertheless, consensus on the safety of same-day discharge after appendectomy has yet to be established [16, 17], and most patients are still hospitalized for 1 or 2 nights after appendectomy for simple appendicitis [5, 18-20]. The aim of this study was to assess the safety of same-day discharge after appendectomy for acute appendicitis by performing a systematic review and critical appraisal of the available literature.

\section{Methods}

\section{Protocol}

A study protocol was established and entered in the International Prospective Register of Systematic Reviews PROSPERO network (registration no. CRD42018115948) [21]. This systematic review was conducted according to the PRISMA guidelines [22]. In addition, the Cochrane Handbook for Systematic Reviews of Interventions [23] and the AMSTAR 2 Checklist were used [24].

\section{Search strategy}

A comprehensive search was performed in EMBASE, Ovid MEDLINE, Web of science, Cochrane Central, Google Scholar, and ClinicalTrials.gov from inception to April 14, 2020. The initial query was developed in consultation with a library scientist. Among other, search terms included "appendicitis,", "appendectomy," "hospital discharge," "ambulatory," "outpatient," and "day case." The complete search strategy is outlined in Online Resource "Appendix A. " The search was limited to articles published in the English language. Manual reference checks were performed in relevant articles.

\section{Study selection}

Studies presenting outcome data for patients with same calendar day discharge (SDD) after appendectomy were eligible. In this study, SDD included ambulatory appendectomy, daycase appendectomy, and any other protocol of discharge on the day of appendectomy without overnight hospital stay [1-3]. The following study types were included: randomized controlled trial, prospective observational (cohort) study, retrospective observational (cohort) study, case-control study, and case series. Studies were included if at least one of the main outcomes was reported. Titles and abstracts were first screened for eligibility. Articles were excluded if the abstract revealed no relevance to the subject or if they concerned one of the following: conservative/nonoperative treatment of appendicitis, case reports, and editorials without evaluation data. Two reviewers (EW and JB) independently assessed all nonduplicate articles for inclusion. Disagreements were resolved via negotiated consensus. Subsequently, full-text articles of potentially eligible studies were reviewed, and a final selection of studies was agreed on. If full-text was unavailable, the corresponding author was contacted to request access. Reasons for exclusion after full-text screening are reported in the flowchart (Fig. 1).

\section{Risk of bias assessment}

Two reviewers independently assessed the risk of bias in each comparative study, using the Risk Of Bias In Non-randomized Studies-of Interventions (ROBINS-I) tool [25]. The ROBINSI tool evaluates the risk of bias in 7 domains: bias due to confounding, bias in selection of participants into the study, bias in classification of interventions, bias due to deviations from intended interventions, bias due to missing data, bias in measurement of outcomes, and bias in selection of the reported results.

\section{Outcomes}

The main outcomes were hospital readmission, complications and unplanned hospital visits within 30 days after appendectomy. Complications were defined as any complication overall or any surgical site complication. Unplanned hospital visits were defined as visits to the Emergency Room (ER) and/or to the outpatient clinic (excluding planned postoperative followup appointments).

Secondary outcomes were (radiological or surgical) reinterventions, length of hospital stay, costs, and treatment satisfaction.

\section{Data extraction and statistical analysis}

Outcome data were extracted as well as data on study period and origin, study design, patient selection, number of patients, characteristics of study group, and follow-up time. Data were collected by one reviewer and verified by another. Outcomes are either displayed as reported originally or calculated from the raw reported data. Uncomplicated acute appendicitis was defined as acute appendicitis without findings of necrosis/ gangrene or perforation, unless otherwise specified.

Only comparative studies were considered for meta-analysis. Assessment of the study characteristics identified three 
Fig. 1 PRISMA flow diagram. From Moher D, Liberati A, Tetzlaff J, Altman DG, The PRISMA Group (2009). Preferred reporting items for systematic reviews and metaAnalyses: the PRISMA statement. PLoS Med 6(7): e1000097. doi:10.1371/ journal.pmed1000097

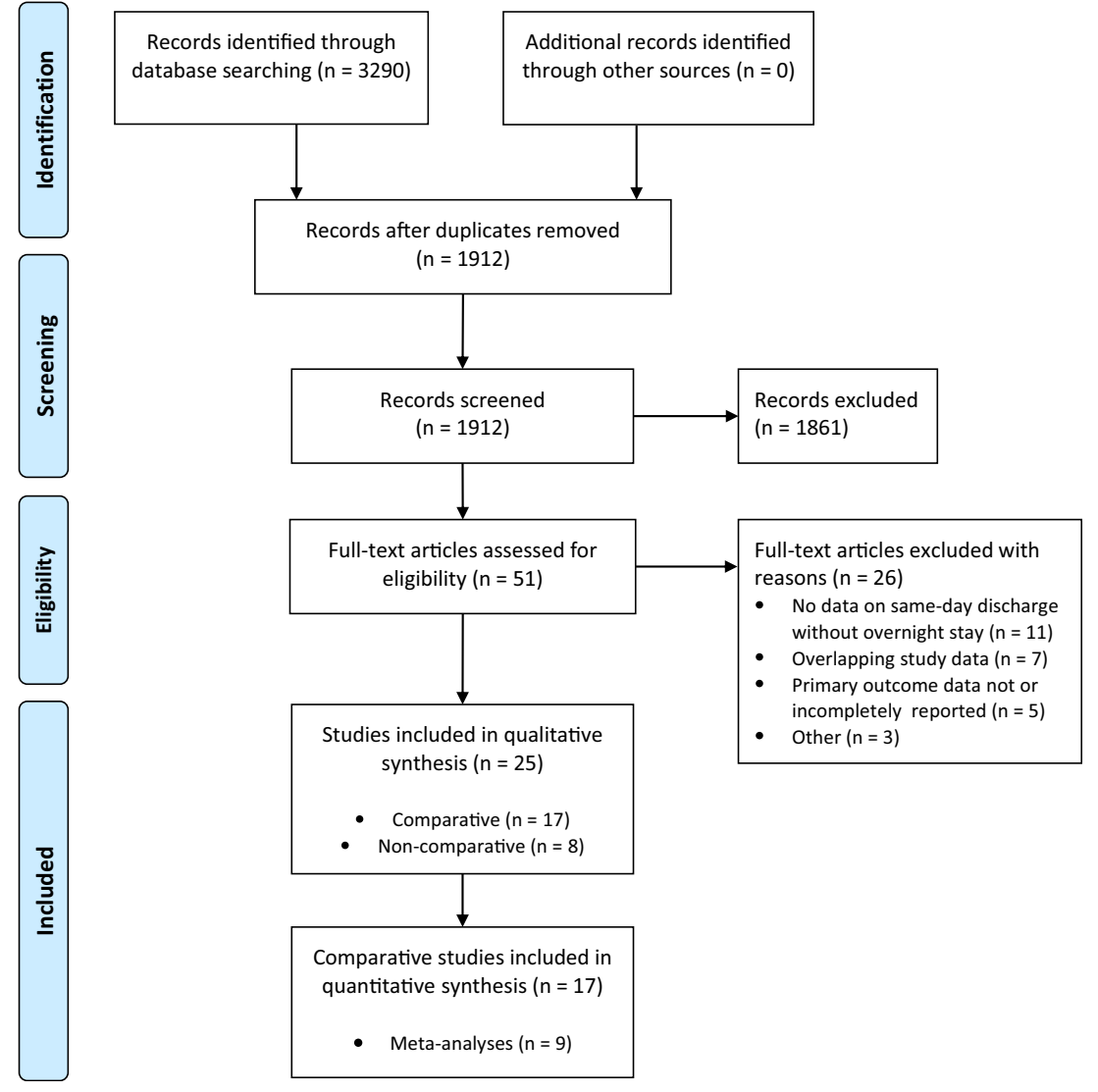

methodological categories. Some studies compared SDD in a prospective cohort with a historical cohort. Three studies compared SDD to discharge on postoperative day (POD) 1 or 2 and excluded patients discharged after 2 days. This was done to exclude patients with prolonged hospital stay due to immediate complications and/or medical reasons. The third category comprises of studies that compared patients with SDD to patients with overnight stay (for one or more nights) during the same study period. This group of studies was felt to be conceptually different from the other studies, since the control groups included patients that stayed overnight for various reasons that may have affected their chance of adverse outcomes: medical reasons (i.e., nausea, pain, comorbidities, complex type appendicitis), social and organizational reasons (i.e., late surgery, home $>1 \mathrm{~h}$ from hospital, no accompanying adult). It was decided to exclude these studies from meta-analysis. The other study categories were considered appropriate for meta-analysis but inappropriate for pooling together due to heterogeneity in study design. Hence, meta-analyses were conducted separately for studies comparing patients in a SDD protocol to historical controls and studies comparing SDD to discharge on POD1-2.

Meta-analyses were performed for the risk ratio (RR) of three outcomes (readmission, complications, and unplanned hospital visits), using a random-effects meta-analysis model.
In this model, the Sidik-Jonkman method was used to estimate the between-study variance $[26,27]$. The $I^{2}$-statistic and Cochran's $Q$ test were used to assess statistical heterogeneity between studies. Meta-analysis was also applied to presented results with adjustment for covariates, based on the published adjusted odds ratios (OR) and confidence intervals in two studies (Cairo et al. adjusted for: age, ASA-class, sex, race, and ethnicity [5]; Grigorian et al. adjusted for age, wound classification, ASA-class, several comorbidities, and steroid use [15]). Results are presented in forest plots. Analyses were performed in $\mathrm{R}$ version 3.5.2 [28].

\section{Results}

\section{Study selection}

Literature search identified 1912 non-duplicate articles. After abstract and full-text review, 25 studies, 17 comparative, and 8 non-comparative observational studies were included. The flowchart of the study selection is presented in Fig. 1. The rate of same-day discharge among the cohorts ranged from 22 to $96 \%$. Ten studies included pediatric patients only and ten studies adults only. Five studies included patients from all ages. 


\section{Comparative studies}

Characteristics of all comparative studies are shown in Table 1, grouped into three categories according to study design. Five studies compared patients in a prospective SDD protocol to patients from a historical control cohort (with a lower percentage of SDD) [12, 29-32]. Three multicenter retrospective studies compared SDD to discharge on POD 1 or 2 at the latest $[5,15,33]$. The remaining nine studies compared successful SDD to overnight hospitalization for one or more nights [7, 9, 34-40]. Overnight hospitalization occurred for varying reasons of medical, social, and organizational nature. Since these factors may well have affected the outcomes of interest, the latter group of studies was excluded from metaanalysis. Variations in cohort selection criteria, discharge criteria, and reasons for failing SDD are further illustrated in supplementary table S1 (Online Resource 'Appendix B').

\section{Risk of bias assessment}

The ROBINS-I results are highlighted in supplementary table S2 (Online Resource 'Appendix B'). The overall risk of bias was considered moderate in five studies, serious in ten studies and critical in two studies.

Table 2 outlines the main outcomes for the comparative studies.

\section{Hospital readmission}

Fifteen studies with varying duration of follow-up reported readmission rates (Table 2). Readmission after SDD ranged from 0 to $4.6 \%$. One study reported a significantly higher readmission rate for the SDD protocol cohort [12]. Metaanalysis with pooled data from four studies comparing readmission rates for SDD protocol patients vs. historical controls demonstrated a RR of 1.47, 95\% CI 0.56 to 3.84 (Fig. 2a). Meta-analysis with pooled data from 3 studies comparing readmission rates for SDD vs. discharge on POD1-2 demonstrated a RR of $0.76,95 \%$ CI 0.67 to 0.88 (Fig. 2b). Metaanalysis with pooled adjusted data from two of the latter studies showed a similar association: OR $0.81,95 \%$ CI 0.68 to 0.97 (Fig. 2c). No statistically significant between-study heterogeneity or between-study variance was observed in any of the meta-analyses ( $I^{2}$ and Cochran's $Q$ results shown in Fig. 2).

\section{Postoperative complications}

All 17 studies reported postoperative complications. Rates varied between $\%$ and 19\% (Table 2). There was inconsistency in the definitions used for complications (table S1, Appendix B). One study reported a significantly higher rate of complications for SDD protocol patients [31]. Meta- analysis with pooled data from five studies comparing complication rates for SDD protocol patients vs. historical controls demonstrated a RR of $1.18,95 \%$ CI 0.73 to 1.91 (Fig. 3a). Meta-analysis with pooled data from 3 studies comparing complication rates for SDD vs. discharge on POD1-2 demonstrated a RR of $0.77,95 \%$ CI 0.65 to 0.90 (Fig. 3b). Metaanalysis with pooled adjusted data from two of the latter studies showed a significant association as well: OR $0.64,95 \% \mathrm{CI}$ 0.42 to 0.97 (Fig. 3c). No statistically significant betweenstudy heterogeneity was observed in any of the metaanalyses ( $I^{2}$ and Cochran's $Q$ results shown in Fig. 3).

\section{Unplanned hospital visits}

Eleven studies described unplanned visits to the hospital, ranging from 0 to $12.6 \%$ after SDD (Table 2). One study found a significantly higher rate for the SDD protocol group [12]. The remaining studies found no difference in the rate of unplanned visits. Meta-analysis with pooled data from three studies comparing complication rate for SDD protocol patients vs. historical controls showed a RR of $1.30,95 \% \mathrm{CI}$ 0.68 to 2.49 (Fig. 4). No statistically significant betweenstudy heterogeneity was observed $\left(I^{2} 53 \%, 95 \%\right.$ CI $0-87 \%$, Cochran's $Q$ test $p=0.12$ ).

\section{Other outcomes}

Reinterventions-Six comparative studies reported reinterventions to some extent, all showing reoperation occurrence below $1 \%$ after SDD $[9,15,30,36,37,40]$. There were no significant differences in reoperation rate between SDD and control group patients (details in Table S3, Appendix B). Another six studies that reported complications, did not present any reintervention in their study cohorts $[7,29,34,35$, $38,39]$.

Length of stay-Thirteen studies reported length of stay, as displayed in Table 1 in hours. Mean postoperative length of stay after SDD ranged from $3.1 \pm 1.4$ [36] to 9.6 (standard deviation not given) [39] h. Nine studies tested for significance, all reporting a statistically significant reduction in LOS for SDD compared to control groups $[7,9,12,30,32$, $35,36,39,40]$.

Costs-Seven studies performed a cost analysis [7, 12, 30, $33,36,38,40]$. Methods of cost analysis were reported in only four studies, and concerned direct hospital-costs, societal costs were outside the scope (details in table S4, Appendix B). All seven studies reported a cost reduction in the SDD group compared to controls, ranging from $\$ 323$ [30] to \$4111 [36]. Three studies showed a statistically significant cost reduction (Table S3, Appendix B).

Treatment satisfaction-Five studies reported treatment satisfaction to some extent $[7,32,35,39,40]$. Various short, non-validated surveys were used at different postoperative 


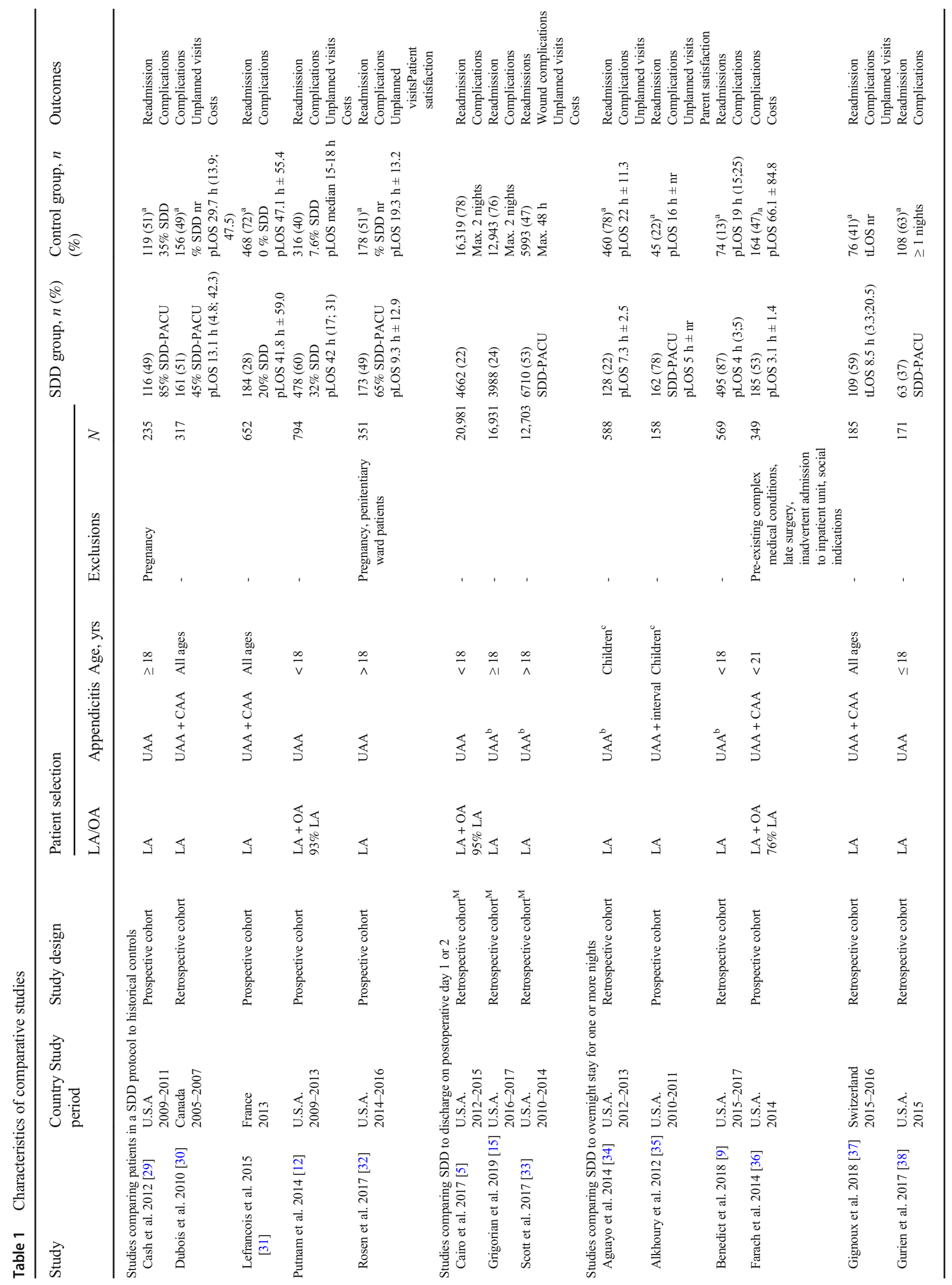




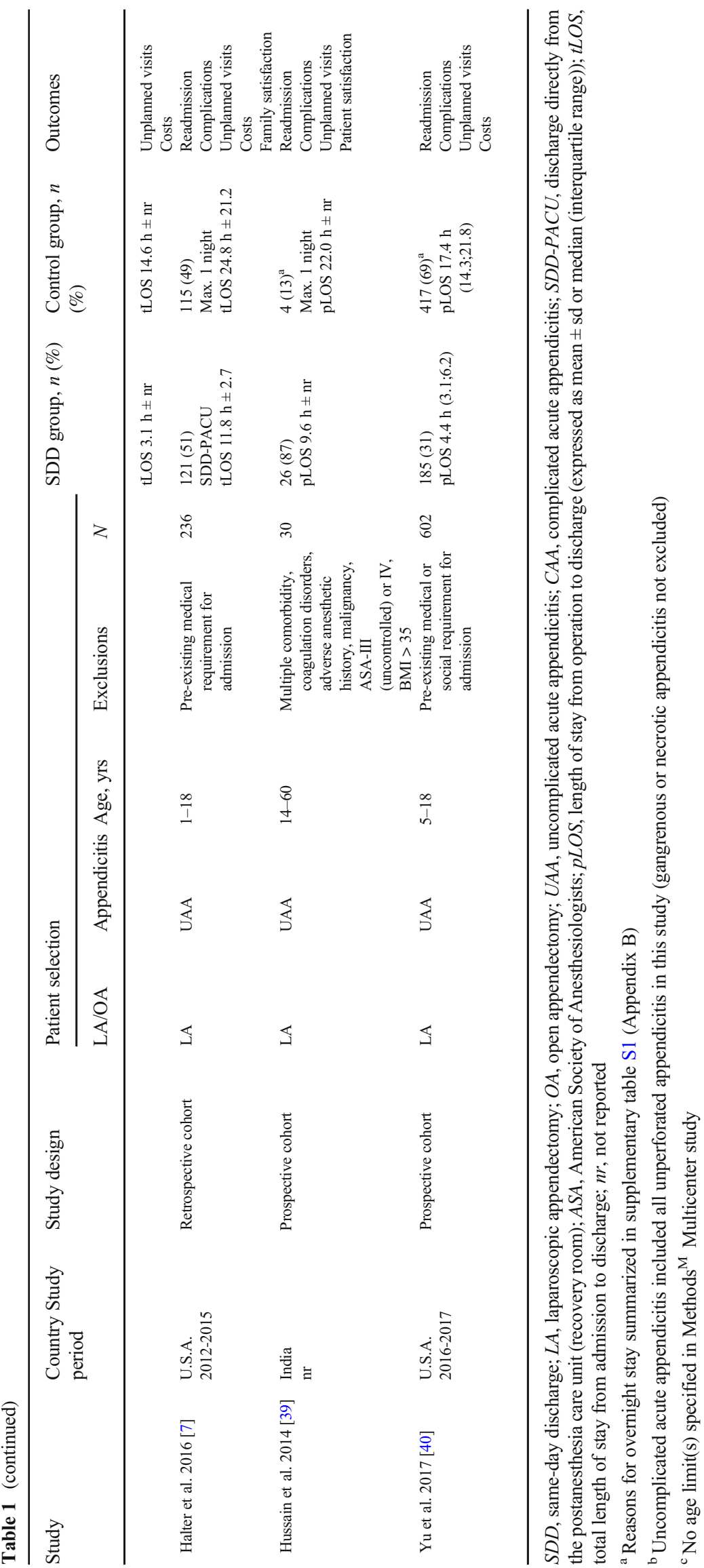




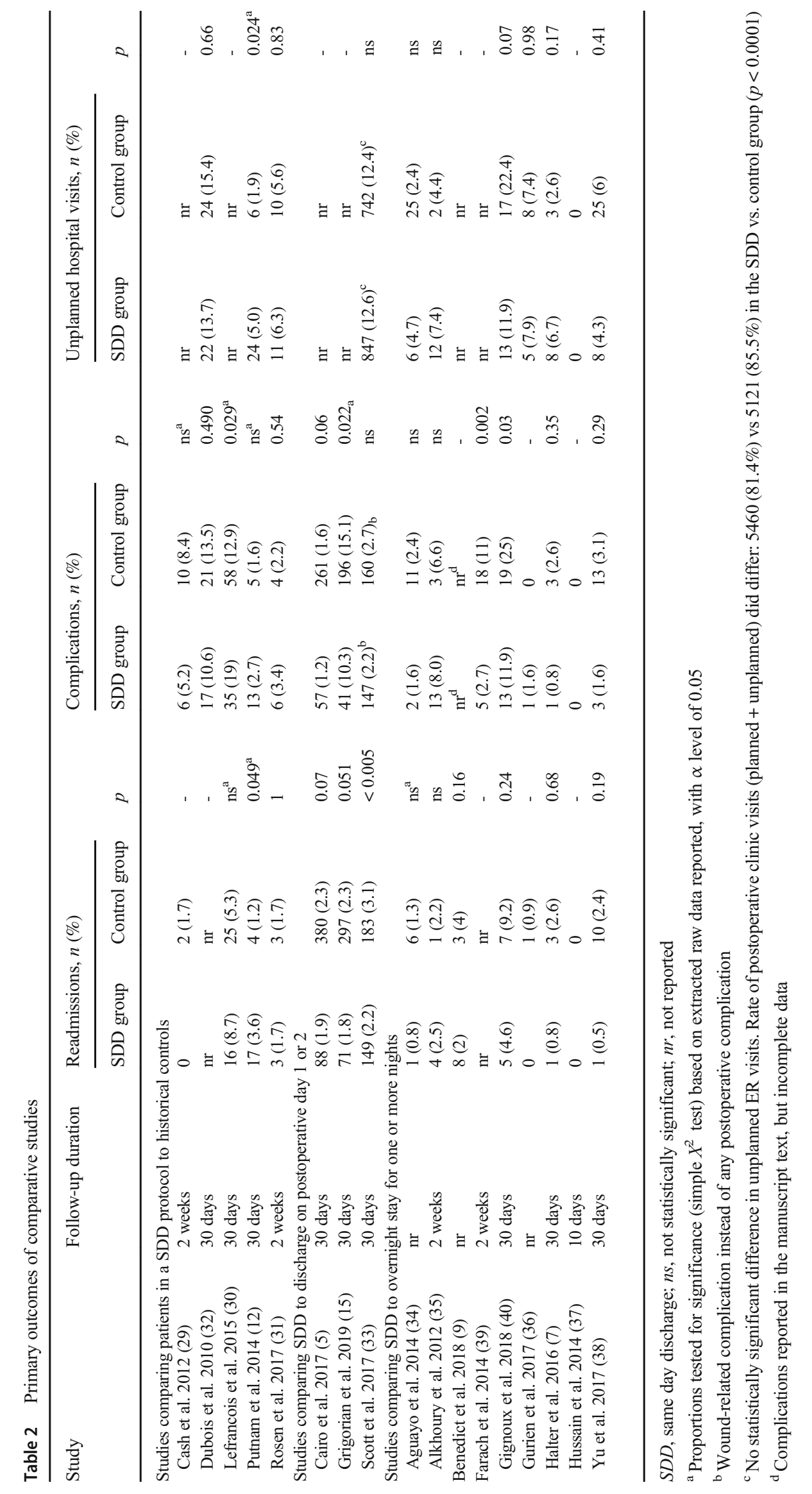


Fig. 2 Meta-analyses on the association between SDD and rate of readmission a

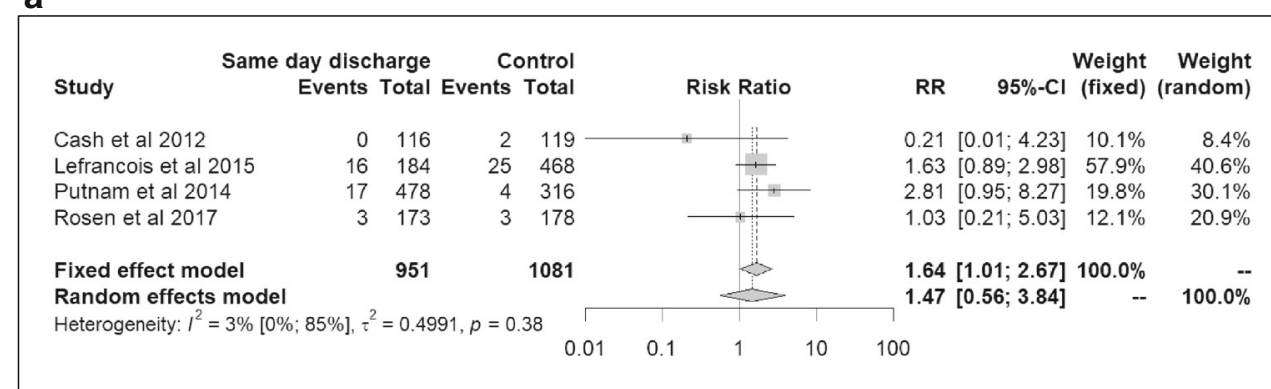

b

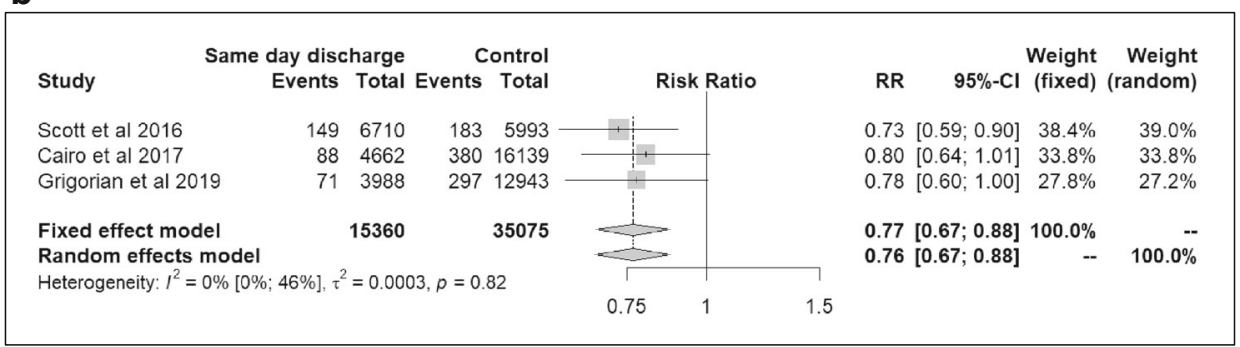

C

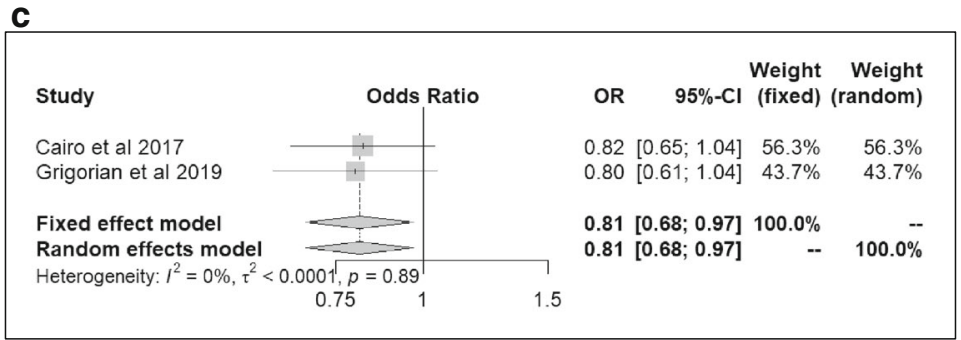

points in time (details in Table S5, Appendix B). Overall, the studies reported high patient satisfaction after SDD. One study presented satisfaction scores for both SDD protocol patients and historical controls and showed no differences [32].

\section{Non-comparative studies}

Eight non-comparative, observational studies reported outcomes after implementation of an SDD protocol [4, 8, 14, 41-45]. Their characteristics and main results are shown in Table 3. Seven studies reported successful SDD in $80 \%$ or more of their selected population. One study reported only $40 \%$ SDD [8]. This study only included patients aged 2-18 years. Reported readmission and complication rates ranged from 0 to $6.9 \%$ and 0 to $12.8 \%$, respectively. Unplanned hospital visits were observed in 8.1 to $13.2 \%$ of patients.

With regard to secondary outcomes: reintervention rates ranged from 0 to $3.6 \%$ in 7 studies (Table S3, Appendix B), none analyzed costs, and only one study evaluated treatment satisfaction and quality of life (Table S5, Appendix B).

\section{Discussion}

This systematic review demonstrated no increased risk of adverse outcomes following same-day discharge (SDD) after appendectomy. Meta-analyses revealed either no significant association between SDD and rates of readmission, complication and unplanned visits, or a statistically signification association in favor of SDD. Due to substantial clinical and methodological between-study heterogeneity, pooling of data for meta-analysis was limited.

Fifteen of the 17 included comparative studies showed no increase in any adverse outcome after SDD. Two studies reported a statistically significant increase in one or two adverse outcomes after SDD. The differences presented may not be clinically relevant. Hence, same-day discharge seems safe and may be encouraged after careful selection of patients. Results on secondary outcomes (very low rate of reinterventions, significantly reduced postoperative length of stay, indication of reduced costs, no indication of reduced treatment satisfaction), further support SDD. If SDD after appendectomy would be applied more frequently in the future, this will likely reduce hospitalization and associated healthcare costs. With the results of this review in mind, it may be of interest to perform 
Fig. 3 Meta-analyses on the association between SDD and rate of complications a

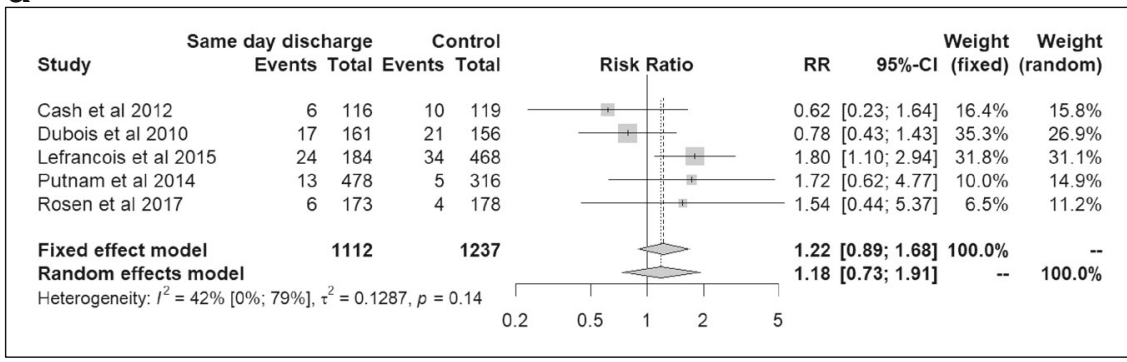

b

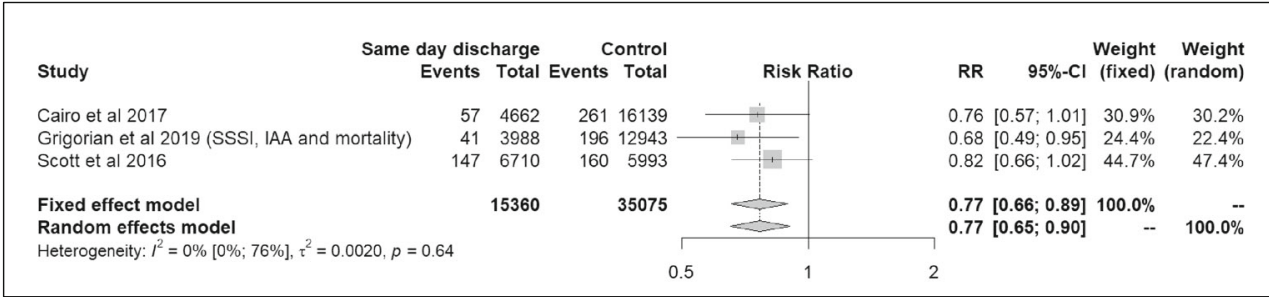

C

\begin{tabular}{|c|c|c|c|c|c|}
\hline Study & Ratio & OR & $95 \%-\mathrm{Cl}$ & $\begin{array}{l}\text { Weight } \\
\text { (fixed) }\end{array}$ & $\begin{array}{r}\text { Weight } \\
\text { (random) }\end{array}$ \\
\hline Cairo et al 2017 & & \multirow{2}{*}{\multicolumn{2}{|c|}{$\begin{array}{l}0.75[0.56 ; 1.01] \\
0.48[0.28 ; 0.82]\end{array}$}} & $76.8 \%$ & $63.2 \%$ \\
\hline Grigorian et al 2019 (SSSI only) & & & & $23.2 \%$ & $36.8 \%$ \\
\hline Fixed effect model & & \multirow{4}{*}{\multicolumn{2}{|c|}{$\begin{array}{l}0.68[0.52 ; 0.88] \\
0.64[0.42 ; 0.97]\end{array}$}} & $100.0 \%$ & -- \\
\hline Random effects model & & & & -- & $100.0 \%$ \\
\hline \multirow{2}{*}{ 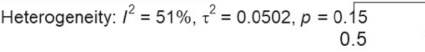 } & & & & & \\
\hline & 1 & & & & \\
\hline
\end{tabular}

appendectomies early during the day, thereby enabling SDD. Protocols designed to facilitate SDD may be helpful to reduce the need for hospital beds and health care workers, especially during the night.

In contrast to previously published reviews, the present study focused on discharge on the same calendar day as the operation and excluded studies that did not explicitly report SDD [15-17]. Sabbagh et al. performed a review on the feasibility of ambulatory surgery ( $<12 \mathrm{~h}$ length of stay) for several gastrointestinal emergencies in adults [16]. Only three of the 12 included studies on early discharge after appendectomy concerned ambulatory surgery, two of which explicitly reported SDD and are therefore included in the present review. The authors concluded that there is probably a place for ambulatory surgery in clinical practice. Cosse et al. conducted a review on the feasibility of day-case appendectomy for acute appendicitis in adults [2]. They included the same studies as Sabbagh et al. as well as a duplicate publication by Cash et al.
$[29,46]$. Seven studies reported day-case appendectomy, defined as $<24 \mathrm{~h}$ length of stay (hence none were included in the present review). The authors stated that day-case appendectomy was safe and feasible, but more prospective studies should be performed before accepting day-case appendectomy as standard care. Genser et al. also reviewed ambulatory appendectomy and included only three studies, all of which are included in the present review as well. They concluded that ambulatory appendectomy for uncomplicated appendicitis is feasible and may be implemented [17]. Most studies included in these reviews were of retrospective nature. Best evidence would come from prospective trials. A randomized study would be ideal but may not be feasible or ethical. TrejoAvila et al. recently published a randomized trial related to this topic [10]. In this study, 108 patients were randomized to an enhanced recovery protocol (ERAS) or standard care. Ambulatory management (defined as postoperative LOS < $12 \mathrm{~h}$ ) was achieved in $90 \%$ in the ERAS group vs. $3.4 \%$ for
Fig. 4 Meta-analysis on the association between SDD and rate of unplanned hospital visits

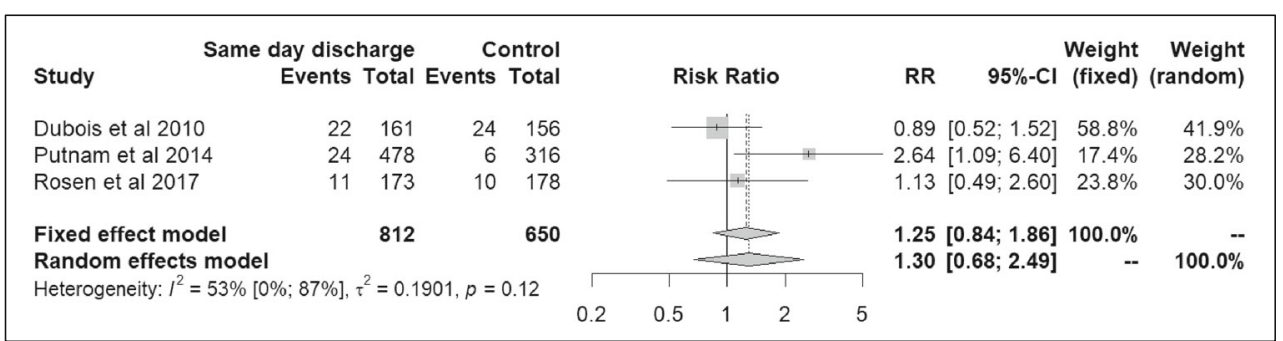




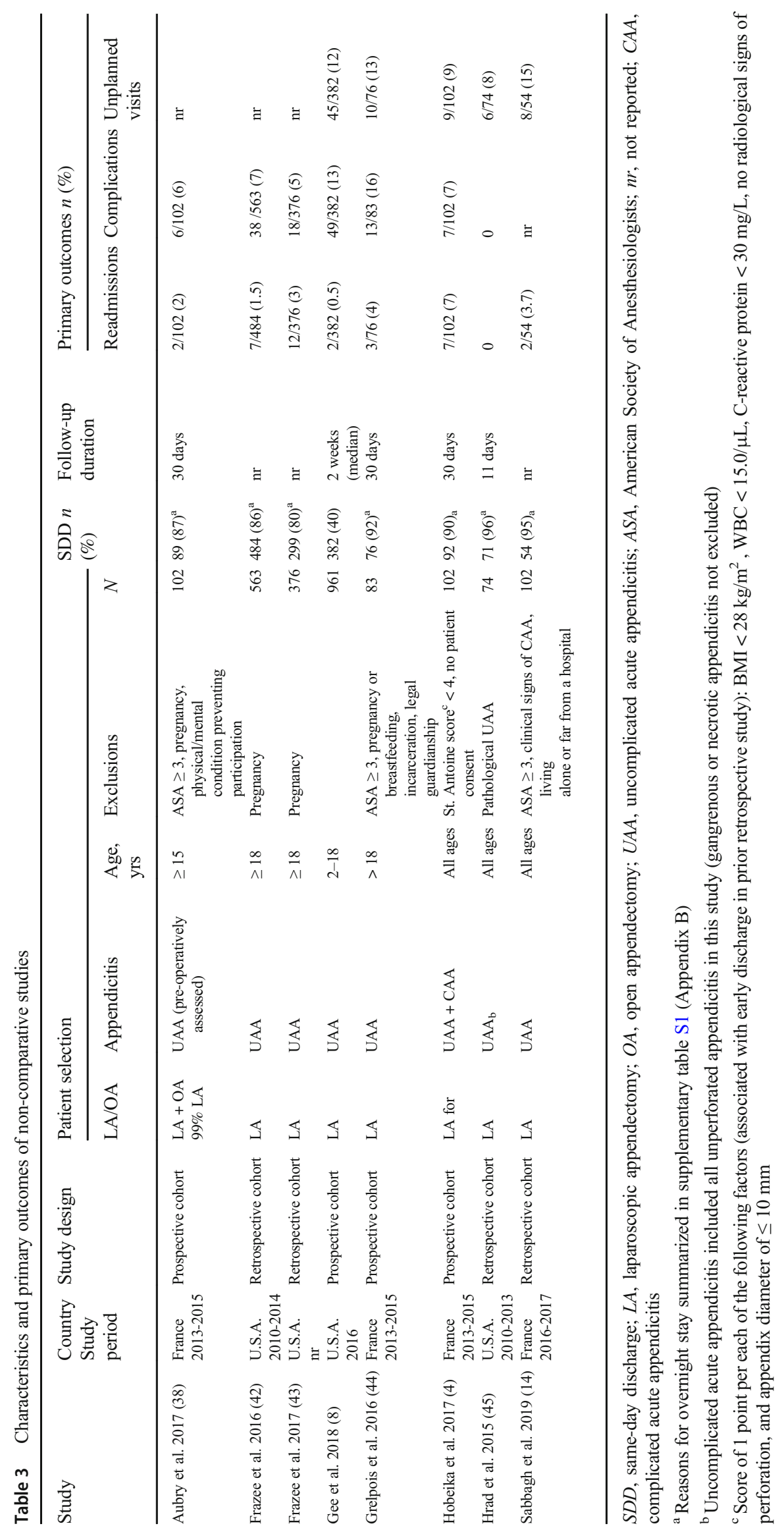


standard care [10]. Though this RCT could not be included in the present review as there was no explicit report of (the proportion of) discharge on the same calendar day, it does support the findings of the present study. The same authors also performed a systematic review and meta-analysis on ambulatory appendectomy for adult patients [13]. The results are in concordance with ours and represent the best currently available evidence on early discharge after appendectomy. Remarkably, many studies have misleading titles: incorporating the words "same-day discharge," "outpatient"and/or "ambulatory," whilst not actually reporting discharge without overnight stay [1]. This was a main reason for excluding full text articles in the present review. Nevertheless, an additional 10 comparative studies were included that were not assessed in the previous reviews, reporting data from both pediatric and adult study populations. Furthermore, eight non-comparative studies were included to summarize evidence on same-day discharge completely. Clinical outcomes after implementation of an SDD protocol in the non-comparative studies were similar to those in the comparative studies.

SDD is feasible and safe in a large proportion of patients. Based on the heterogeneous sample of studies in this review, it is difficult to establish one optimum set of patient selection and discharge criteria for SDD. Selection criteria used in most studies are uncomplicated/unperforated appendicitis and laparoscopic surgery. Twenty-one of the 25 studies in this review excluded open procedures from their cohort. In four studies that included both laparoscopic and open procedures, the proportion of open procedures was low and no separate outcome data were available. Hence, no conclusions can be drawn concerning the safety of SDD after open appendectomy. Both adult and pediatric patients can be considered eligible for SDD after laparoscopic appendectomy. Exclusion of ASA-class III-IV and pregnant patients was often applied as well and seems appropriate. Discharge criteria should entail normal vital signs, ability to tolerate oral intake, ability to ambulate and pain controlled by oral analgesics. Ultimately, the goal will not be to discharge all patients on the day of appendectomy, but to improve treatment efficiency by facilitating same-day discharge in a larger proportion of eligible patients. A same-day discharge protocol preferably entails a concise set of eligibility criteria that can be assessed preoperatively for the most part. Patients discharged this quickly after surgery should be well informed of relevant signs and symptoms of complications. And adequate (reporting of) follow-up is essential to evaluate the effects of adapting such a protocol.

This study has some limitations. Only non-randomized observational studies were included, which are prone to bias, e.g., due to confounding and selective reporting of results. Meta-analysis was only justified for a limited number of studies. Due to the small number of studies in the meta-analyses, funnel plots for identifying publication bias were not felt to be of added value and statistical between-study heterogeneity (though not observed) cannot be ruled out. Many of the included studies compared SDD patients to a non-matched control group of patients with overnight stay (determined by different medical, social and organizational reasons). Moreover, there was substantial clinical heterogeneity (varying patient selection criteria) as well as methodological heterogeneity (varying study design) among the studies. Lastly, variation in duration of follow-up may have resulted in underreported events. Nonetheless, strengths of the present study are its systematic and extensive nature. A preregistered protocol was adhered to, and the PRISMA guidelines were followed [14], resulting in a large number of recently published studies that was included.

\section{Conclusion}

Current literature provides no indication that same-day discharge is unsafe. Adequate patient selection may be the key to stimulate same-day discharge. It appears safe for most patients undergoing laparoscopic appendectomy for uncomplicated acute appendicitis that meet discharge criteria. Data on costs and treatment satisfaction presented in this review were rather limited. Further implementation of same-day discharge after appendectomy may lower expenses and enhance patient satisfaction.

Supplementary Information The online version contains supplementary material available at https://doi.org/10.1007/s00384-021-03872-3.

Acknowledgements The authors thank Wichor Bramer of the Medical Library of the Erasmus MC-University Medical Centre for developing search strategies.

Author contribution All authors contributed to the study conception and design. EW and JB screened and selected studies, assessed risk of bias, and extracted data. Statistical analysis was performed by JR in cooperation with EW. EW and JB first drafted this manuscript. All authors reviewed and approved the final manuscript.

Funding EW and $\mathrm{AB}$ were supported by a grant from ZonMw (The Netherlands Organization for Health Research and Development; project no. 848015008). ZonMw had no role in the design or conduct of this study.

Data availability and materials availability All study data and material are available in the (supplementary) tables and in the original studies.

\section{Declarations}

Competing interests The authors declare no competing interests.

Open Access This article is licensed under a Creative Commons Attribution 4.0 International License, which permits use, sharing, 
adaptation, distribution and reproduction in any medium or format, as long as you give appropriate credit to the original author(s) and the source, provide a link to the Creative Commons licence, and indicate if changes were made. The images or other third party material in this article are included in the article's Creative Commons licence, unless indicated otherwise in a credit line to the material. If material is not included in the article's Creative Commons licence and your intended use is not permitted by statutory regulation or exceeds the permitted use, you will need to obtain permission directly from the copyright holder. To view a copy of this licence, visit http://creativecommons.org/licenses/by/4.0/.

\section{References}

1. Genser L, Vons C (2015) Can abdominal surgical emergencies be treated in an ambulatory setting. J Visc Surg 152(6):S81-S89. https://doi.org/10.1016/j.jviscsurg.2015.09.015

2. Cosse C, Sabbagh C, Grelpois G, Brehant O, Regimbeau JM (2014) Day case appendectomy in adults: a review. Int J Surg 12(7):640-644. https://doi.org/10.1016/j.ijsu.2014.05.072

3. Sabbagh C, Cosse C, Dupont H, Ntouba A, Lion T, Regimbeau JM (2014) Ambulatory management of gastrointestinal emergencies: what are the current literature data. J Visc Surg 151(1):23-27. https://doi.org/10.1016/j.jviscsurg.2013.10.007

4. Hobeika C, Hor T, Chereau N, Laforest A, Bachmann R, Sourouille I, Chafai N, Parc Y, Beaussier M, Lefèvre JH (2017) Day surgery for acute appendicitis in adults: a prospective series of 102 patients. Surg Laparoscopy Endosc Percutaneous Tech 27(3):158-162. https://doi.org/10.1097/sle.0000000000000394

5. Cairo SB, Raval MV, Browne M, Meyers H, Rothstein DH (2017) Association of same-day discharge with hospital readmission after appendectomy in pediatric patients. JAMA Surg 152(12):11061112. https://doi.org/10.1001/jamasurg.2017.2221

6. Grewal H, Sweat J, Vazquez WD (2004) Laparoscopic appendectomy in children can be done as a fast-track or same-day surgery. JSLS 8(2):151-154

7. Halter JM, Mallory B, Neilson IR, Langer M (2016) Same-day discharge following laparoscopic appendectomy for uncomplicated acute appendicitis as a measure of quality in the pediatric population. J Laparoendosc Adv Surg Techn 26(4):309-313. https://doi. org/10.1089/lap.2016.0093

8. Gee K, Ngo S, Burkhalter L, Beres AL (2018) Safety and feasibility of same-day discharge for uncomplicated appendicitis: a prospective cohort study. J Pediatr Surg 53(5):988-990. https://doi.org/10. 1016/j.jpedsurg.2018.02.031

9. Benedict LA, Sujka J, Sobrino J, Aguayo P, St. Peter SD, Oyetunji TA (2018) Same-day discharge for nonperforated appendicitis in children: an updated institutional protocol. J Surg Res 232:346350. https://doi.org/10.1016/j.jss.2018.06.057

10. Trejo-Ávila ME, Romero-Loera S, Cárdenas-Lailson E, BlasFranco M, Delano-Alonso R, Valenzuela-Salazar C, MorenoPortillo M (2019) Enhanced recovery after surgery protocol allows ambulatory laparoscopic appendectomy in uncomplicated acute appendicitis: a prospective, randomized trial. Surg Endosc 33(2):429436. https://doi.org/10.1007/s00464-018-6315-9

11. Rochon RM, Gimon T, Buie WD, Brar MS, Dixon E, MacLean AR (2019) Expedited discharge in uncomplicated acute appendicitis: decreasing the length of stay while maintaining quality. Am J Surg 217(5):830-833. https://doi.org/10.1016/j.amjsurg.2019.03. 007

12. Putnam LR, Levy SM, Johnson E, Williams K, Taylor K, Kao LS, Lally KP, Tsao K (2014) Impact of a 24-hour discharge pathway on outcomes of pediatric appendectomy. Surgery 156(2):455-461. https://doi.org/10.1016/j.surg.2014.03.030
13. Trejo-Avila M, Cárdenas-Lailson E, Valenzuela-Salazar C, Herrera-Esquivel J, Moreno-Portillo M (2019) Ambulatory versus conventional laparoscopic appendectomy: a systematic review and meta-analysis. Int J Color Dis 34(8):1359-1368. https://doi.org/10. 1007/s00384-019-03341-y

14. Sabbagh C, Masseline L, Grelpois G, Ntouba A, Dembinski J, Regimbeau JM (2019) Management of uncomplicated acute appendicitis as day case surgery: can outcomes of a prospective study be reproduced in real life. J Am Coll Surg 229(3):277-285. https://doi. org/10.1016/j.jamcollsurg.2019.04.031

15. Grigorian A, Kuza CM, Schubl SD, Nguyen NT, de Virgilio C, Kim D, Lekawa M, Nahmias J (2019) Same-day Discharge after non-perforated laparoscopic appendectomy is safe. J Investig Surg: 1-6. https://doi.org/10.1080/08941939.2019.1630065

16. Bhangu A, Søreide K, Di Saverio S, Assarsson JH, Drake FT (2015) Acute appendicitis: modern understanding of pathogenesis, diagnosis, and management. Lancet 386(10000):1278-1287. https://doi.org/10.1016/S0140-6736(15)00275-5

17. Stewart B, Khanduri P, McCord C, Ohene-Yeboah M, Uranues S, Vega Rivera F, Mock C (2014) Global disease burden of conditions requiring emergency surgery. Br J Surg 101(1):e9-e22. https://doi. org/10.1002/bjs.9329

18. Ceresoli M, Zucchi A, Allievi N, Harbi A, Pisano M, Montori G, Heyer A, Nita GE, Ansaloni L, Coccolini F (2016) Acute appendicitis: epidemiology, treatment and outcomes- analysis of 16544 consecutive cases. World J Gastrointest Surg 8(10):693-699. https://doi.org/10.4240/wjgs.v8.i10.693

19. Sartelli M, Baiocchi GL, Di Saverio S, Ferrara F, Labricciosa FM, Ansaloni L, Coccolini F, Vijayan D, Abbas A, Abongwa HK, Agboola J, Ahmed A, Akhmeteli L, Akkapulu N, Akkucuk S, Altintoprak F, Andreiev AL, Anyfantakis D, Atanasov B, Bala M, Balalis D, Baraket O, Bellanova G, Beltran M, Melo RB, Bini R, Bouliaris K, Brunelli D, Castillo A, Catani M, Che Jusoh A, Chichom-Mefire A, Cocorullo G, Coimbra R, Colak E, Costa S, Das K, Delibegovic S, Demetrashvili Z, Di Carlo I, Kiseleva N, El Zalabany T, Faro M, Ferreira M, Fraga GP, Gachabayov M, Ghnnam WM, Gimenez Maurel T, Gkiokas G, Gomes CA, Griffiths E, Guner A, Gupta S, Hecker A, Hirano ES, Hodonou A, Hutan M, Ioannidis O, Isik A, Ivakhov G, Jain S, Jokubauskas M, Karamarkovic A, Kauhanen S, Kaushik R, Kavalakat A, Kenig J, Khokha V, Khor D, Kim D, Kim JI, Kong V, Lasithiotakis K, Leao P, Leon M, Litvin A, Lohsiriwat V, Lopez-Tomassetti Fernandez E, Lostoridis E, Maciel J, Major P, Dimova A, Manatakis D, Marinis A, Martinez-Perez A, Marwah S, McFarlane M, Mesina C, Pedziwiatr M, Michalopoulos N, Misiakos E, Mohamedahmed A, Moldovanu R, Montori G, Mysore Narayana R, Negoi I, Nikolopoulos I, Novelli G, Novikovs V, Olaoye I, Omari A, Ordonez CA, Ouadii M, Ozkan Z, Pal A, Palini GM, Partecke LI, Pata F, Pedziwiatr M, Pereira Junior GA, Pintar T, Pisarska M, Ploneda-Valencia CF, Pouggouras K, Prabhu V, Ramakrishnapillai P, Regimbeau JM, Reitz M, Rios-Cruz D, Saar S, Sakakushev B, Seretis C, Sazhin A, Shelat V, Skrovina M, Smirnov D, Spyropoulos C, Strzalka M, Talving P, Teixeira Gonsaga RA, Theobald G, Tomadze G, Torba M, Trana C, Ulrych J, Uzunoglu MY, Vasilescu A, Occhionorelli S, Venara A, Vereczkei A, Vettoretto N, Vlad N, Waledziak M, Yilmaz TU, Yuan KC, Yunfeng C, Zilinskas J, Grelpois G, Catena F (2018) Prospective Observational Study on acute Appendicitis Worldwide (POSAW). World J Emerg Surg 13:19. https://doi.org/10.1186/s13017-018-0179-0179

20. Worni M, Ostbye T, Gandhi M, Rajgor D, Shah J, Shah A, Pietrobon R, Jacobs DO, Guller U (2012) Laparoscopic appendectomy outcomes on the weekend and during the week are no different: a national study of 151,774 patients. World J Surg 36(7):15271533. https://doi.org/10.1007/s00268-012-1550-z 
21. Safety of same-day discharge after appendectomy. (2018) PROSPERO International Prospective register of systematic reviews. CRD42018115948. https://www.crd.york.ac.uk/prospero/ display_record.php?RecordID $=115948$. Accessed December, 10 2018

22. Moher D, Liberati A, Tetzlaff J, Altman DG, Group P (2009) Preferred reporting items for systematic reviews and meta-analyses: the PRISMA statement. Ann Intern Med 151(4):264-269 W264

23. Higgins JPT, Green S eds (2011) Cochrane handbook for systematic reviews of interventions. The Cochrane Collaboration. http:// handbook-5-1.cochrane.org/. Accessed December 14, 20182011

24. Shea BJ, Reeves BC, Wells G, Thuku M, Hamel C, Moran J, Moher D, Tugwell P, Welch V, Kristjansson E, Henry DA (2017) AMSTAR 2: a critical appraisal tool for systematic reviews that include randomised or non-randomised studies of healthcare interventions, or both. BMJ 358:j4008. https://doi.org/10.1136/bmj. j4008

25. Sterne JA, Hernan MA, Reeves BC, Savovic J, Berkman ND, Viswanathan M, Henry D, Altman DG, Ansari MT, Boutron I, Carpenter JR, Chan AW, Churchill R, Deeks JJ, Hrobjartsson A, Kirkham J, Juni P, Loke YK, Pigott TD, Ramsay CR, Regidor D, Rothstein HR, Sandhu L, Santaguida PL, Schunemann HJ, Shea B, Shrier I, Tugwell P, Turner L, Valentine JC, Waddington H, Waters E, Wells GA, Whiting PF, Higgins JP (2016) ROBINS-I: a tool for assessing risk of bias in non-randomised studies of interventions. BMJ 355:i4919. https://doi.org/10.1136/bmj.i4919

26. Sidik K, Jonkman JN (2007) A comparison of heterogeneity variance estimators in combining results of studies. Stat Med 26(9): 1964-1981. https://doi.org/10.1002/sim.2688

27. Mantel N, Haenszel W (1959) Statistical aspects of the analysis of data from retrospective studies of disease. J Natl Cancer Inst 22(4): 719-748

28. R Core Team (2018). R: A language and environment for statistical computing. R Foundation for Statistical Computing, Vienna, Austria. URL: https://www.R-project.org/

29. Cash CL, Frazee RC, Abernathy SW, Childs EW, Davis ML, Hendricks JC, Smith RW (2012) A prospective treatment protocol for outpatient laparoscopic appendectomy for acute appendicitis. J Am Coll Surg 215(1):101-105. https://doi.org/10.1016/j. jamcollsurg.2012.02.024

30. Dubois L, Vogt KN, Davies W, Schlachta CM (2010) Impact of an outpatient appendectomy protocol on clinical outcomes and cost: acase-control study. J Am Coll Surg 211(6):731-737. https://doi. org/10.1016/j.jamcollsurg.2010.07.017

31. Lefrancois M, Lefevre JH, Chafai N, Pitel S, Kerger L, Agostini J, Canard G, Tiret E (2015) Management of acute appendicitis in ambulatory surgery: is it possible? How to select patients? Ann Surg 261(6):1167-1172. https://doi.org/10.1097/sla. 0000000000000795

32. Rosen DR, Inaba K, Oh PJ, Gutierrez AC, Strumwasser AM, Biswas S, Cala M, Ault GT (2017) Outpatient laparoscopic appendectomy: feasible in a public county hospital. J Am Coll Surg 224(5):862-867. https://doi.org/10.1016/j.jamcollsurg.2017.02. 004

33. Scott A, Shekherdimian S, Rouch JD, Sacks GD, Dawes AJ, Lui WY, Bridges L, Heisler T, Crain SR, Cheung MW, Aboulian A (2017) Same-day discharge in laparoscopic acute non-perforated appendectomy. J Am Coll Surg 224(1):43-48. https://doi.org/10. 1016/j.jamcollsurg.2016.10.026

34. Aguayo P, Alemayehu H, Desai AA, Fraser JD, St. Peter SD (2014) Initial experience with same day discharge after laparoscopic appendectomy for nonperforated appendicitis. J Surg Res 190(1): 93-97. https://doi.org/10.1016/j.jss.2014.03.012

35. Alkhoury F, Malvezzi L, Knight CG, Diana J, Pasaron R, Mora J, Aserlind A, Stylianos S, Burnweit C (2012) Routine same-day discharge after acute or interval appendectomy in children: a prospective study. Arch Surg 147(5):443-446. https://doi.org/10.1001/ archsurg.2012.132

36. Farach SM, Danielson PD, Walford NE, Harmel RP, Chandler NM (2014) Same-day discharge after appendectomy results in cost savings and improved efficiency. Am Surg 80(8):787-791

37. Gignoux B, Blanchet MC, Lanz T, Vulliez A, Saffarini M, Bothorel H, Robert M, Frering V (2018) Should ambulatory appendectomy become the standard treatment for acute appendicitis. World $\mathrm{J}$ Emerg Surg 13:28. https://doi.org/10.1186/s13017-018-0191-4

38. Gurien LA, Burford JM, Bonasso PC, Dassinger MS (2017) Resource savings and outcomes associated with outpatient laparoscopic appendectomy for nonperforated appendicitis. J Pediatr Surg 52(11):1760-1763. https://doi.org/10.1016/j.jpedsurg.2017.03.039

39. Hussain A, Singh S, Singh Ahi K, Singh M (2014) Status of day care laparoscopic appendectomy in developing countries. Int Sch Res Notices 2014:502786

40. Yu YR, Smith CM, Ceyanes KK, Naik-Mathuria BJ, Shah SR, Vogel AM, Carberry KE, Nuchtern JG, Lopez ME (2018) A prospective same day discharge protocol for pediatric appendicitis: adding value to a common surgical condition. J Pediatr Surg 53(1):36-41. https://doi.org/10.1016/j.jpedsurg.2017.10.011

41. Aubry A, Saget A, Manceau G, Faron M, Wagner M, Tresallet C, Riou B, Lucidarme O, le Saché F, Karoui M (2017) Outpatient appendectomy in an emergency outpatient surgery unit $24 \mathrm{~h}$ a day: an intention-to-treat analysis of 194 patients. World J Surg 41(10):2471-2479. https://doi.org/10.1007/s00268-017-4034-3

42. Frazee RC, Abernathy SW, Isbell CL, Isbell T, Regner JL, Smith RD (2016) Outpatient laparoscopic appendectomy: is it time to end the discussion. J Am Coll Surg 222(4):473-477. https://doi.org/10. 1016/j.jamcollsurg.2015.12.053

43. Frazee R, Burlew CC, Regner J, McIntyre R, Peltz E, Cribari C, Dunn J, Butler L, Reckard P, Dissanaike S, Karimi K, Behnfield C, Melo N, Margulies D (2017) Outpatient laparoscopic appendectomy can be successfully performed for uncomplicated appendicitis: a Southwestern Surgical Congress multicenter trial. Am J Surg 214(6):1007-1009. https://doi.org/10.1016/j.amjsurg.2017.08.029

44. Grelpois G, Sabbagh C, Cosse C, Robert B, Chapuis-Roux E, Ntouba A, Lion T, Regimbeau JM (2016) Management of uncomplicated acute appendicitis as day case surgery: feasibility and a critical analysis of exclusion criteria and treatment failure. J Am Coll Surg 223(5):694-703. https://doi.org/10.1016/j.jamcollsurg. 2016.08.004

45. Hrad V, Waisbren SJ (2015) Results of immediate discharge from postanesthesia care unit to home after laparoscopic appendectomy for acute nonperforated appendicitis. Surg Laparoscopy Endosc Percutaneous Tech 25(4):343-346. https://doi.org/10.1097/sle. 0000000000000170

46. Cash CL, Frazee RC, Smith R, Davis ML, Hendricks MJC, Childs EW, Abernathy SW (2012) Outpatient laparoscopic appendectomy for acute appendicitis. Am Surg 78(2):213-215

Publisher's note Springer Nature remains neutral with regard to jurisdictional claims in published maps and institutional affiliations. 